\title{
Whole-exome sequencing of a patient with severe and complex hemostatic abnormalities reveals a possible contributing frameshift mutation in C3AR1
}

\author{
Eva Leinøe, ${ }^{1}$ Ove Juul Nielsen, ${ }^{1}$ Lars Jønson, ${ }^{2}$ and Maria Rossing ${ }^{2}$ \\ ${ }^{1}$ Department of Hematology, Rigshospitalet, University of Copenhagen, DK-2100 Copenhagen, Denmark; \\ ${ }^{2}$ Center for Genomic Medicine, Rigshospitalet, University of Copenhagen, DK-2100 Copenhagen, Denmark
}

\begin{abstract}
The increasing availability of genome-wide analysis has made it possible to rapidly sequence the exome of patients with undiagnosed or unresolved medical conditions. Here, we present the case of a 64-yr-old male patient with schistocytes in the peripheral blood smear and a complex and life-threatening coagulation disorder causing recurrent venous thromboembolic events, severe thrombocytopenia, and subdural hematomas. Whole-exome sequencing revealed a frameshift mutation (C3AR1 c.355-356dup, p.Asp119Alafs*19) resulting in a premature stop codon in C3AR1 (Complement Component 3a Receptor 1). Based on this finding, atypical hemolytic uremic syndrome was suspected because of a genetic predisposition, and a targeted treatment regime with eculizumab was initiated. Life-threatening hemostatic abnormalities would most likely have persisted had it not been for the implementation of whole-exome sequencing in this particular clinical setting.
\end{abstract}

Corresponding author: caroline. maria.rossing@regionh.dk

(C) 2016 Leinøe et al. This article is distributed under the terms of the Creative Commons Attribution-NonCommercial License, which permits reuse and redistribution, except for commercial purposes, provided that the original author and source are credited.

Ontology terms: acute disseminated intravascular coagulation; recurrent deep vein thrombosis

Published by Cold Spring Harbor Laboratory Press

doi: $10.1101 /$ mcs.a000828

\section{INTRODUCTION}

Atypical hemolytic uremic syndrome (aHUS) is an inherited, rare, progressive, relapsing, and life-threatening disease caused by genetic abnormalities in the complement system. The condition is characterized by chronic or intermittent uncontrolled activation of the complement system resulting in systemic microangiopathy and formation of blood clots in small vessels. Loss-of-function or gain-of-function mutations in components of the alternative pathway account for $60 \%-70 \%$ of all cases of aHUS (Mele et al. 2014; Tsai 2014). Clinical features include consumptive thrombocytopenia, microangiopathic hemolytic anemia, microvascular thrombosis, kidney dysfunction, and hypertension. The chronic hemolysis may predispose the patients to venous thromboembolisms (VTEs) because of nitric oxide scavenging by free hemoglobin (Villagra et al. 2007), and evidence also links complement activation to thrombosis (Conway 2015).

The increasing availability of high-throughput genetic analyses by next-generation sequencing has made it possible to rapidly sequence the exome of patients with undiagnosed or unresolved medical conditions. Generally, sequencing confirms or excludes a particular suspected genetic predisposition. Sometimes sequencing provides new insights or clinically actionable information, and in many cases sequencing yields variants 
of unknown significance. Especially among patients with a suspected inherited coagulation disorder, the identification of genetic defects is increasing rapidly (Leo et al. 2015; Westbury et al. 2015). Here, we describe an example of a whole-exome sequencing (WES)-enabled possible diagnosis of aHUS due to a genetic predisposition in an undiagnosed patient with complex and life-threatening abnormal hemostasis and facilitated a targeted treatment regime.

\section{RESULTS}

\section{Case Presentation}

We present a 64-yr-old male patient with arterial hypertension and four previously recurrent VTE episodes with onset at the age of 48 . The patient has no children, and no familial history of VTE, thrombocytopenia, or bleeding was found. Repeated thrombophilia investigations were negative, and there were no clinical indications of an underlying malignancy. Although the patient was treated with anticoagulants, he developed recurrent VTEs; therefore, the level of international normalized ratio was kept between 3.0 and 3.5. The patient also had a history of chronic thrombocytopenia with an element of pseudothrombocytopenia, as the platelet count was consistently higher when measured in citrated whole blood with added paraformaldehyde (PFA). Consequently, all platelet counts were measured in triplicats (EDTA, citrate, and citrate with PFA) and agglutination occurred in EDTA and citrate tubes. Therefore, in the following paragraphs, all mentioned platelet counts are counted in citrate tubes with added PFA. The patient developed a subdural hematoma during an episode with a very low platelet count of $30 \times 10^{9} / \mathrm{L}$ and therefore warfarin treatment was discontinued. Successive treatments with prednisone, dexamethasone, intravenous immunoglobulins, romiplostim, and rituximab were initiated on suspicion of immune thrombocytopenic purpura; however, a substantial increase in the platelet count was not achieved. Consecutive bone marrow examinations performed in 2002, 2009, and 2015 showed no dysplastic features. During a period with headache and severe thrombocytopenia $\left(9 \times 10^{9} / \mathrm{L}\right)$, a cerebral computed tomography (CT) scan revealed progression of the persistent subdural hematoma and a new subdural hematoma in the opposite hemisphere (Fig. 1A). The patient did not present any neurological deficits at the time and the Glasgow coma score was 15. Treatment with platelet transfusions and tranexamic acid was initiated. During the next 24 $h$, he developed epistaxis, increasing headache, dyspnea, and swelling of the left crus. A CT scan showed progression of the right subdural hematoma with centerline displacement (Fig. 1B). At the same time, a thoracic CT scan verified multiple pulmonary emboli (Fig. 1C). Furthermore, another deep venous thrombosis was diagnosed in the left femoral vein by ultrasound. To guide the hemostatic and anticoagulant treatment in this complex situation, thromboelastography (TEG) was used. D-dimer was increased $(23 \mathrm{mg}$ fibrinogen-equivalent units (FEU)/L, reference: $<0.5 \mathrm{mg} F E U / L$ ), whereas activated partial thromboplastin time and international normalized ratio were in the normal range. Treatment with fibrinogen concentrate (RiaSTAP) and platelet concentrates was initiated, leading to a rise in platelet counts, improvement in the strength of the clot evaluated by TEG, and allowing the initiation of low-molecular-weight heparin treatment in prophylactic doses. A peripheral blood smear was performed because of transfusion-requiring anemia, demonstrating $4 \%$ schistocytes (Fig. 2A). The morphology of the platelets was variable, showing both microthrombocytes and giant platelets but no platelet aggregates. Plasma concentration of ADAMTS13 was normal. Repeated plasmapheresis prompted normalization of platelet count, peripheral blood smear, and all TEG parameters. As hemoglobin was normalized and the frequency of plasmapheresis was reduced, the platelet count decreased again and the patient developed renewed symptoms of pulmonary emboli despite therapeutic anticoagulation treatment. 

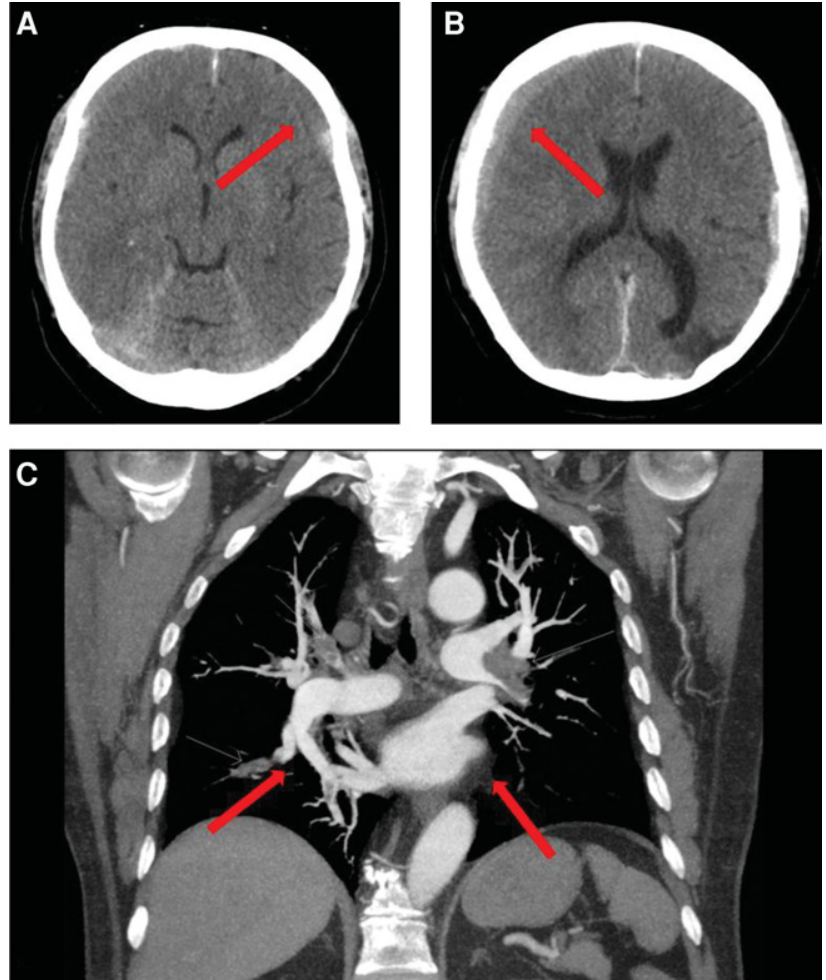

Figure 1. Computed tomography (CT) images of cerebrum showing left $(A)$ and right $(B)$ subdural hematomas. (C) Coronal section of a thoracic CT scan showing bilateral pulmonary emboli.

A pulmonary CT scan showed a saddle embolus of the pulmonary arteries, and there were clinical signs of progression of deep venous thrombosis in the left leg. Platelet counts fell to $50 \times 10^{9} / \mathrm{L}$ and schistocytes were again present in the peripheral blood smear. Additional antithrombotic treatment with aspirin was initiated combined with daily plasmapheresis. A repeated cerebral CT scan demonstrated progression of the subdural hematoma. At this stage, WES was performed to test for inherited dysfibrinogenemia, thrombocytopathia, and thrombotic microangiopathy (TAM). Congenital dysfibrinogenemia was suspected because of low fibrinogen activity and prolonged thrombin time. Thrombotic microangiopathia was suspected because of schistocytes, nonimmune hemolysis, and slightly decreased

A

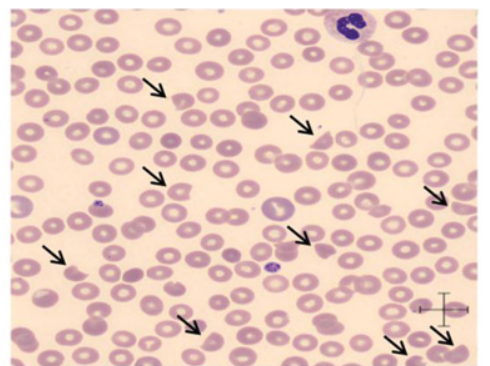

B

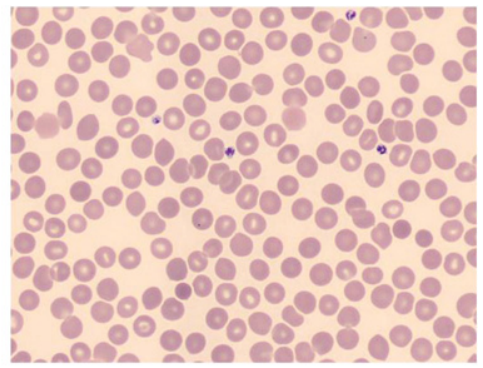

Figure 2. (A) Peripheral blood smear with schistocytes before treatment with eculizumab. (B) Peripheral blood smear without schistocytes after eculizumab treatment. 
Table 1. C3AR1 (NM_004054.2) variant table

\begin{tabular}{|c|c|c|c|c|c|c|c|c|}
\hline Gene & Chromosome & $\begin{array}{l}\text { HGVS DNA } \\
\text { reference }\end{array}$ & $\begin{array}{l}\text { HGVS protein } \\
\text { reference }\end{array}$ & $\begin{array}{l}\text { Variant } \\
\text { type }\end{array}$ & $\begin{array}{l}\text { Predicted } \\
\text { effect }\end{array}$ & dbSNP ID & Genotype & Ethnicity \\
\hline C3AR1 & $12: 8212426$ & hg19 & NP_004045.1 & Duplication & Pathogenic & rs553595145 & Heterozygous & Caucasian \\
\hline
\end{tabular}

HGVS, Human Genome Variation Society; dbSNP, Database for Short Genetic Variations.

kidney function (P-creatinine $132 \mathrm{mmol} / \mathrm{L}$; ref. 60-105 mmol/L). A renal biopsy was not performed because of the patient's severe bleeding tendency.

\section{Genomic Analysis}

Genomic DNA from the patient was analyzed by WES. As no mutations were detected in the genes encoding fibrinogen ( $F G A$, fibrinogen a chain; $F G B$, fibrinogen $\beta$ chain; and $F G G$, fibrinogen $\gamma$ chain), the initial working diagnosis of dysfibrinogenemia was ruled out. To exclude additional thrombophilic defects, antithrombin (SERPINC1), protein $\mathrm{S}(P R O S 1)$, protein $\mathrm{C}$ (PROC), factor $\mathrm{V}(F 5)$, factor II (F2), and thrombomodulin were analyzed without identifying any predisposing variants in these genes. However, a heterozygous insertion in C3AR1 (Complement Component 3a Receptor 1), c.355-356dup, p. Asp119Alafs*19 was identified (Table 1; Fig. 3). The variant causes a premature stop at codon 170 of complement component 3a receptor 1. The c.355-356dup variant in C3AR1 was listed with a frequency of $0.02 \%$ in the background population in the ExAC (Exome Aggregation Consortium) Browser Beta database. C3AR1 encodes the C3a anaphylatoxin chemotactic receptor involved in the alternative pathway of the complement system (Ricklin and Lambris 2007). Additional complement genes (CFH, CFI, C3, CFB, and CD46) were included in the WES data analysis, including manually inspecting the last three exons of $C F H$, to rule out any false-negative result.

This specific variant had previously been reported as deleterious in a single patient with aHUS (Bu et al. 2014). The study by Bu et al. was based on 36 patients with sporadic aHUS, recognized by acute renal failure, thrombocytopenia, and microangiopathic hemolytic anemia after excluding a causal Shiga-toxigenic Escherichia coli infection. Upon recognition of a pathogenic variant in an aHUS-associated gene in combination with the patient's symptoms, the diagnosis of aHUS was suspected and targeted treatment with monoclonal antibodies was initiated.

\section{Immediate Treatment Outcome}

Until the recent availability of eculizumab-a monoclonal antibody that is a terminal complement inhibitor-aHUS carried a significant morbidity and mortality rate with $25 \%$ of patients dying of the disease and $50 \%$ of patients progressing to end-stage kidney failure (Noris and Remuzzi 2009). Treatment of aHUS with eculizumab was initiated, whereas plasmapheresis treatments were scaled down. The patient efficiently increased his platelet counts to $>100 \times 10^{9} / \mathrm{L}$, and no schistocytes were present in the peripheral blood smear following $12 \mathrm{wk}$ of eculizumab treatment (Figs. 2B and 4). We analyzed frozen samples taken before initiation of plasmapheresis, as well as before initiation of eculizumab, and found that complement activity (measured by the alternative pathways) was indeed significantly reduced. As expected, all complement pathways (lectin, classical, and alternative) were reduced following treatment with eculizumab. A weakness of this retrospective analysis is that the sample had been frozen at $-20^{\circ} \mathrm{C}$ instead of the recommended $-80^{\circ} \mathrm{C}$. Six months on from the initiation of eculizumab along with warfarin treatment, the patient's condition is stable with no further thrombosis, no signs of hemolysis, normal platelet count (in citrate 


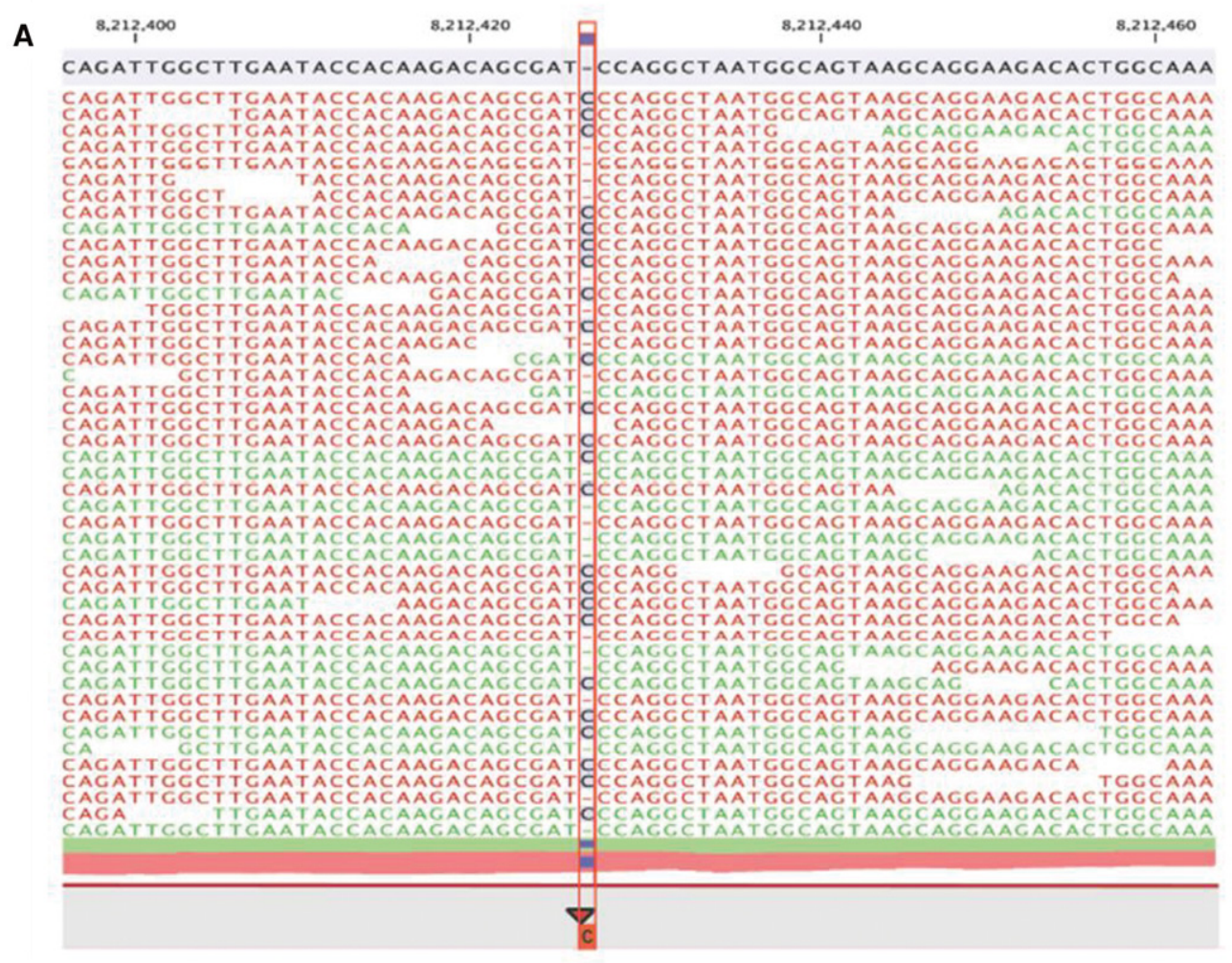

B

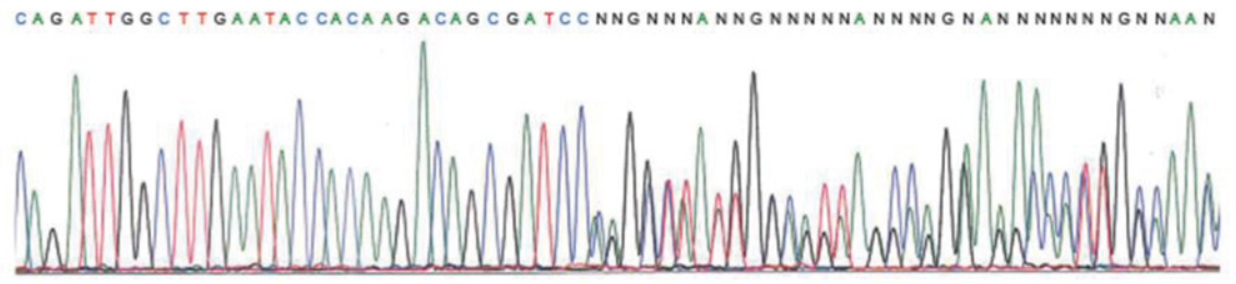

Figure 3. Frameshift mutation in C3AR1 c.355-356dup, p.Asp119Alafs*19. (A) Read mapping in CLC Genomics Workbench to hg19 (+ strand) showing the insertion on Chr 12:8212426. (B) Forward reads are shown in green and reverse reads in red. SeqScape illustration showing the Sanger sequencing of the polymerase chain reaction product covering the mutation.

added with PFA tubes), and no bleeding episodes. The pseudo thrombocytopenia persists with agglutination of platelets in EDTA and citrate tubes.

\section{DISCUSSION}

Initially, a diagnosis of immune thrombocytopenic purpura or dysfibrinogenemia was suspected in a critically ill 64-yr-old male who presented with complex hemostatic abnormalities involving recurrent VTE, severe thrombocytopenia, and progressive subdural hematomas. Biochemical evidence of microangiopathic hemolytic anemia prompted suspicion of a complement disease. By taking advantage of WES we identified a frameshift mutation in the complement C3a receptor (C3AR1) gene and thereby diagnosed a critically ill patient with 


\begin{tabular}{|c|c|c|c|c|c|c|c|c|c|c|c|c|}
\hline & & & & & & $\begin{array}{l}\text { lood } \\
\text { ample fo }\end{array}$ & & $\begin{array}{l}\text { WES fin } \\
\text { report }\end{array}$ & & $\begin{array}{l}\text { culizuma } \\
\text { reatmen }\end{array}$ & & \\
\hline Date & $\begin{array}{c}2009 \\
-14\end{array}$ & D1 & D23 & D33 & D35 & D57 & D65 & D79 & D84 & D125 & D132 & D297 \\
\hline $\begin{array}{l}\text { Schistocyte } \\
\mathrm{s}(\%)\end{array}$ & & & & & 4 & 3-4 & & & $0-1$ & & & \\
\hline $\begin{array}{l}\text { Platelets } \\
\text { (x10 }\end{array}$ & $\approx 40$ & 4 & 9 & 16 & 94 & 55 & 278 & 272 & 152 & 99 & 116 & 178 \\
\hline Event & & SDH & SDH & $\begin{array}{l}\text { SDH } \\
\text { progres } \\
\text { sion, } \\
\text { PE, } \\
\text { bilater } \\
\text { al DVT }\end{array}$ & $\begin{array}{c}\text { PE; } \\
\text { Saddle } \\
\text { embol } \\
\text { us }\end{array}$ & $\begin{array}{l}\text { SDH } \\
\text { progres } \\
\text { sion }\end{array}$ & & & & & & \\
\hline
\end{tabular}

Figure 4. Time line of clinical events and diagnostic milestones depicting the time of recovery after causal diagnosis by whole-exome sequencing (WES) and initialization of targeted treatment. DVT, deep venous thrombosis; $\mathrm{PE}$, pulmonary embolism; $\mathrm{SDH}$, subdural hematoma.

aHUS. C3AR1 is a cognate G-protein-coupled receptor for activated complement factor C3a. Supporting the potentially pathogenic significance of the C3AR1 mutation found in our patient, an identical mutation was recently reported in a single patient also suffering from aHUS (Bu et al. 2014). Because the C3AR1 mutation is listed with a frequency of $0.02 \%$ (29 individuals), and functional studies of the C3A receptor 1 were not performed, we cannot claim that this mutation is definitely pathogenic. It is not possible to support a clear Mendelian inheritance, where a single pathogenic allele is sufficient to explain the phenotype, based only on our patient case. Although, atypical HUS most often presents itself in childhood ( $<18 \mathrm{yr}$ ), penetrance of aHUS is unpredictable, and the onset of symptoms can vary from pediatric to elderly patients (Fremeaux-Bacchi et al. 2006). aHUS may not occur until middle age because of incomplete penetrance, suggesting that a triggering stimulus is required for the disease to manifest (Esparza-Gordillo et al. 2005). Thus, it seems that especially in elderly patients, aHUS appears to be a "two-hit" disease, where a putative trigger, such as inflammation, autoantibodies, or genetic modifiers, increases complement activation (Brodsky 2015).

At the age of 64, our patient presented with microangiopathic hemolytic anemia, thrombocytopenia, and marginally impaired renal function. Approximately $20 \%$ of patients have a progressive onset with subclinical anemia and fluctuating thrombocytopenia for months, whereas renal function is preserved and normal at diagnosis (Mele et al. 2014). In retrospect, the early symptoms of aHUS appeared at a much earlier age, because our patient had a history of recurrent VTE and hypertension (Scully and Goodship 2014). We speculate that the venous thrombotic events were triggered by continuous low-grade hemolysis, as our patient had mild chronic anemia. The patient was also tested for thrombophilia, which came back negative. Chronic hemolytic anemia induces a procoagulant state through mitigating nitric oxide scavenging by red blood cells (L'Acqua et al. 2015); this may have been the cause of the thrombophilic state of the patient. Furthermore, our patient suffered from progressive microangiopathic hemolysis, which could have been triggered by withdrawal of anticoagulant treatment. In patients with thrombotic microangiopathy, formation of fibrin- and platelet-rich thrombi occurs in the microcirculation, obstructing vessel lumina and leading to endorgan ischemia and infarction. Warfarin treatment may have prevented the formation of fibrin plugs in the microcirculation and fulminant aHUS in our patient for many years. Anticoagulant prophylactic treatment has been proposed for certain patients with hemolytic 
disorders, but there is currently no evidence to define the optimal regimens or groups at high risk (L'Acqua et al. 2015). The C3AR1 frameshift mutation discribed here may manifest with a more varied phenotype than commonly seen aHUS, characterized by lack of kidney failure but severe thrombosis. The WES approach was useful to identify this nonclassic disease presentation and initiate an effective treatment.

Until the introduction of eculizumab, plasmapheresis was considered the "gold standard" for management of aHUS (Taylor et al. 2010). There is no obvious reason why plasmapheresis was effective in normalizing platelet count and microangiopathic haemolysis in our patient, because the C3AR1 mutation is cell-based. No functional studies were performed to examine receptor status; thus, it remains unclear whether the mutation confers reduced receptor-mediated clearance of activated complement components. Plasma transfusions contain endothelial protective molecules (e.g., Antithrombin, Protein C, TFPI), which may lead to stabilization of the activated endothelium; also plasma transfusions replace depleted complement regulators, whereas plasmapheresis remove activated complement components.

Eculizumab is a humanized monoclonal antibody that is a terminal complement inhibitor and was approved for the treatment of aHUS in 2011. It is not yet clear how long a patient with aHUS should be treated with eculizumab. The patient continues to receive treatment with anticoagulation and eculizumab because he has presented multiple and life-threatening episodes of bleedings and VTEs, as this has been a highly efficient treatment of his suspected complement receptor mutation-associated coagulation abnormalities. The continuous treatment with eculizumab, as well as other prescription drugs, will be evaluated during follow-up visits. In conclusion, we detected a possible pathogenic mutation in C3AR1, also reported in 29 individuals in ExAC, in a patient with complex and life-threatening hemostatic abnormalities. However, the functional complement tests and the positive response to eculizumab treatment correspond to a diagnosis of aHUS.

\section{METHODS}

\section{Blood Sample/DNA Extraction/Whole-Exome Sequencing}

Genomic DNA was purified from whole blood using a QIAamp DNA Mini Kit (QIAGEN) according to the manufacturer's instructions. For enrichment of the exome and capturing of 50 $\mathrm{Mb}$ of the coding region, we used a SureSelect All Exon Kit v5 (Agilent). Illumina's HiSeq 2500 platform was used for sequencing. In brief, $1 \mu \mathrm{g}$ of genomic DNA was fractionated on a Covaris S2 to an average size of $250 \mathrm{bp}$. Trimming, 3'-adenylation, and ligation of Illumina-compatible KAPA library DNA adaptors (Roche) were performed on an SPRI-TE nucleic acid extractor using SPRIworks Fragment Library Cartridges I (Beckman Coulter) with a size selection of 200-400 bp. Sequencing was performed on the HiSeq 2500 as paired-end sequencing, $2 \times 101$ bases, resulting in approximately 100 million paired end reads. Sequencing data were processed using CASAVA-1.8.2. Trimming of the last 3 ' base, mapping, and variant calling were performed using CLC Genomics Workbench (QIAGEN). The exome was covered with an average coverage of $145 \times$, where $97.6 \%$ was covered with at least $10 \times$ (Table 2). Variants were called with a minimum of $10 \times$ coverage and exported as .vcf format with approximately 220,000 variants prior to upload in Ingenuity Variant Analysis (QIAGEN). Common single-nucleotide polymorphisms were subtracted, and only variants in genes related to bleeding, thrombocytopathy, thrombopenia, dysfibrinogenemia, thrombotic microangiopathies, and complement activation were examined. The insertion was verified by Sanger sequencing on an ABI 3730 DNA Analyzer (Applied Biosystems) using DNA purified from a second blood sample and the following oligonucleotides for amplification and sequencing, 5'-GGACAGTGAACACAATTTGGTTCC-3' and 5'- 
Competing Interest Statement

The authors have declared no competing interest.

Received December 6, 2015; accepted in revised form April 7, 2016
Table 2. Sequencing coverage

\begin{tabular}{|c|c|c|c|c|c|c|}
\hline Sample & $\begin{array}{l}\text { Percentage } \\
\text { of reads } \\
\text { aligned }\end{array}$ & $\begin{array}{l}\text { Average } \\
\text { read } \\
\text { coverage }\end{array}$ & $\begin{array}{c}\text { Number of } \\
\text { target regions } \\
\text { based on } \\
\text { Ensembl_v74 }\end{array}$ & $\begin{array}{l}\text { Percentage of } \\
\text { Ensembl_v74 } \\
\text { with } \geq 10 \text {-fold } \\
\text { coverage }\end{array}$ & $\begin{array}{l}\text { Percentage of } \\
\text { Ensembl_v74 } \\
\text { with } \geq 25 \text {-fold } \\
\text { coverage }\end{array}$ & $\begin{array}{c}\text { Percentage } \\
\text { of C } 3 A R 1 \\
\text { sites (1449 } \\
\text { bases) with } \\
\geq 116 \times \\
\text { coverage }\end{array}$ \\
\hline Proband & 94 & $145 x$ & 95.304 & 97.6 & 95.7 & 100 \\
\hline
\end{tabular}

Sequencing coverage information for the exome-sequenced patient. The 50-Mbp target region of Agilent's SureSelect v5 was used to calculate average coverage. Consensus coding sequence from Ensembl_v74.

CTGGATAATCTAATGAGCT GG-3'. The C3AR1 mutations are numbered according to the guidelines of the Human Genome Variation Society (http://www.hgvs.org/mutnomen) using National Center for Biotechnology Information (NCBI) Reference Sequence NM_004054.2.

\section{ADDITIONAL INFORMATION}

\section{Data Deposition and Access}

FASTQ files are available at the European Nucleotide Archive (http://www.ebi.ac.uk/ena) under accession number PRJEB12648. The variant is accessible at ClinVar (http://www.ncbi. nlm.nih.gov/clinvar/) under accession number SCV000264331.

\section{Ethics Statement}

Informed and signed consent was obtained for the analysis performed and publication of the results. Likewise, informed and signed consent was obtained with regard to treatment procedures. The patient is included in a larger study regarding WES of patients with suspected genetic predisposition to bleeding. The project is approved by the Research Ethics Committees of the Capital Region of Denmark (\#H-15011677).

\section{Acknowledgments}

We thank Miriam Yan Juk Guo and Christina Ringø for excellent laboratory assistance.

\section{Author Contributions}

E.L. and O.J.N contributed to patient recruitment and phenotyping. M.R. and L.J contributed to sequence data analysis, interpretation, and validation. E.L. and M.R contributed to preparation of the manuscript. All authors contributed to the reviewing of the final version.

\section{REFERENCES}

Brodsky RA. 2015. Complement in hemolytic anemia. Blood 126: 2459-2465.

Bu F, Maga T, Meyer NC, Wang K, Thomas CP, et al. 2014. Comprehensive genetic analysis of complement and coagulation genes in atypical hemolytic uremic syndrome. J Am Soc Nephrol 25: 55-64.

Conway EM. 2015. Reincarnation of ancient links between coagulation and complement. J Thromb Haemost 13(Suppl 1): S121-S132.

Esparza-Gordillo J, Goicoechea de Jorge E, Buil A, Carreras Berges L, López-Trascasa M, Sánchez-Corral P, Ródriguez de Córdoba S. 2005. Predisposition to atypical hemolytic uremic syndrome involves the concurrence of different susceptibility alleles in the regulators of complement activation gene cluster in $1 \mathrm{q} 32$. Hum Mol Genet 14: 703-712. 
Fremeaux-Bacchi V, Moulton EA, Kavanagh D, Dragon-Durey MA, Blouin J, Caudy A, Arzouk N, Cleper R, Francois M, Guest G, et al. 2006. Genetic and functional analyses of membrane cofactor protein (CD46) mutations in atypical hemolytic uremic syndrome. J Am Soc Nephrol 17: 2017-2025.

L'Acqua C, Bandyopadhyay S, Francis RO, McMahon DJ, Nellis M, Sheth S, Kernie SG, Brittenham GM, Spitalnik SL, Hod EA. 2015. Red blood cell transfusion is associated with increased hemolysis and an acute phase response in a subset of critically ill children. Am J Hematol 90: 915-920.

Leo VC, Morgan NV, Bem D, Jones ML, Lowe GC, Lordkipanidzé M, Drake S, Simpson MA, Gissen P, Mumford A, et al. 2015. Use of next-generation sequencing and candidate gene analysis to identify underlying defects in patients with inherited platelet function disorders. J Thromb Haemost 13: 643-650.

Mele C, Remuzzi G, Noris M. 2014. Hemolytic uremic syndrome. Semin Immunopathol 36: 399-420.

Noris M, Remuzzi G. 2009. Atypical hemolytic-uremic syndrome. N Engl J Med 361: 1676-1687.

Ricklin D, Lambris JD. 2007. Complement-targeted therapeutics. Nat Biotechnol 25: 1265-1275.

Scully M, Goodship T. 2014. How I treat thrombotic thrombocytopenic purpura and atypical haemolytic uraemic syndrome. Br J Haematol 164: 759-766.

Taylor CM, Machin S, Wigmore SJ, Goodship TH. 2010. Clinical practice guidelines for the management of atypical haemolytic uraemic syndrome in the United Kingdom. Br J Haematol 148: 37-47.

Tsai HM. 2014. A mechanistic approach to the diagnosis and management of atypical hemolytic uremic syndrome. Transfus Med Rev 28: 187-197.

Villagra J, Shiva S, Hunter LA, Machado RF, Gladwin MT, Kato GJ. 2007. Platelet activation in patients with sickle disease, hemolysis-associated pulmonary hypertension, and nitric oxide scavenging by cell-free hemoglobin. Blood 110: 2166-2172.

Westbury SK, Turro E, Greene D, Lentaigne C, Kelly AM, Bariana TK, Simeoni I, Pilois X, Attwood A, Austin S, et al. 2015. Human phenotype ontology annotation and cluster analysis to unravel genetic defects in 707 cases with unexplained bleeding and platelet disorders. Genome Med 7: 36. 


\section{COLD SPRING HARBOR Molecular Case Studies}

\section{Whole-exome sequencing of a patient with severe and complex hemostatic abnormalities reveals a possible contributing frameshift mutation in C3AR1}

Eva Leinøe, Ove Juul Nielsen, Lars Jønson, et al.

Cold Spring Harb Mol Case Stud 2016, 2: a000828 originally published online April 18, 2016

Access the most recent version at doi:10.1101/mcs.a000828

\begin{aligned} & \hline References $\begin{array}{l}\text { This article cites } 15 \text { articles, } 4 \text { of which can be accessed free at: } \\ \text { http://molecularcasestudies.cshlp.org/content/2/4/a000828.full.htmI\#ref-list-1 }\end{array} \\ &$ License $\begin{array}{l}\text { This article is distributed under the terms of the Creative Commons } \\ \text { Attribution-NonCommercial License, which permits reuse and redistribution, except } \\ \text { for commercial purposes, provided that the original author and source are credited. } \\ \text { Email Alerting } \\ \text { Service }\end{array} \\ & \begin{array}{c}\text { Receive free email alerts when new articles cite this article - sign up in the box at the } \\ \text { top right corner of the article or click here. }\end{array} \\ &$\hline\end{aligned}

